\title{
Study of Densification, Microstructure, and Mechanical Properties in WC-Based Hardmetals Bonded with High and Medium Entropy Alloys
}

M.A. Ruiz-Esparza-Rodriguez ${ }^{1}$, C.G. Garay-Reyes ${ }^{1}$, J.C. Guía-Tello ${ }^{1}$, H. M. Medrano-Prieto ${ }^{2}$, Ivanovich Estrada $^{3}$ and R. Martínez-Sánchez ${ }^{1}$

${ }^{1}$ Centro de Investigación en Materiales Avanzados (CIMAV), Laboratorio Nacional de Nanotecnología, Chihuahua, Chihuahua, México, ${ }^{2}$ Universidad Autónoma de Ciudad Juárez (UACJ), Ciudad Juárez, Chihuahua, Mexico, ${ }^{3}$ Centro de Investigacion en Materiales Avanzados SC, Chihuahua, Chihuahua, Mexico

Usually, for cemented carbides, cobalt is used as a binder in concentration between 15 and 20 wt. \% [1]. However, the recently developed high entropy alloys, which are characterized by having excellent mechanical properties due to its excellent thermodynamic stability, and allows obtaining BCC, FCC or BCC and FCC structures open a wide field of study for cemented carbides since that the cobalt can be replaced with such alloys [2]. Thus, this work focuses on evaluating the densification, microstructure, and mechanical behavior of high (HEA) and medium (MEA) entropy alloys as substitutes of cobalt in cemented carbides.

High (CoCrFeMnNi) and medium (CoCrNi) entropy alloys were synthesized by mechanical alloying (MA). The powders were used had a purity of $99.95 \%$. A high-energy mill Spex 8000 was used to fabricate MEA and HEA for $10 \mathrm{~h}$ of milling time. Subsequently, the compounds WC-HEA and WC-MEA were obtained after alloying them were for $2 \mathrm{~h}$ milling time in mill above mentioned and the concentration variations were determined in 80, 85, 90 and 95\% WC (wt. \%). WC container and Ar atmosphere were used during the MA process to avoid contamination in the compounds, powder mass $8.5 \mathrm{~g}$, and a ball-topowder weight ratio of 5:1 were the process conditions used. A hydraulic press was used for the compaction whit a pressure of $1.56 \mathrm{GPa}$ for 5 minutes, and the sintering process was maintained at $1300^{\circ} \mathrm{C}$ for 1,3 and $5 \mathrm{~h}$ in a continuum vacuum. Finally, the density of the samples was calculated using the Archimedes method in analytical balance Sartorius CP2250, microstructural characterization, up in a scanning electron microscope HITACHI SU3500 and Vickers microhardness was evaluated in LM300 AT tester.

Figure 1 shows SEM-SE micrographs and a densification analysis of the WC-HEA and WC-MEA compounds. An increase in the homogeneity of the microstructure increasing sintering time was observed. In addition and higher densification in the samples was obtained to a low concentration of WC. Figure 2 shows the hardness behavior obtained in WC-HEA and WC-MEA compounds, where a gradual increase in the function of the WC concentration was observed, contrary to the behavior of compound densification in increasing the amount of binder. 
a) WC-MEA

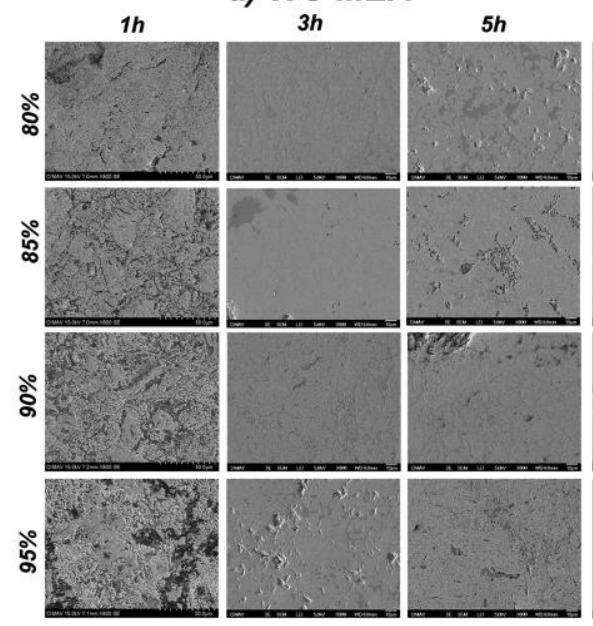

b) WC-HEA

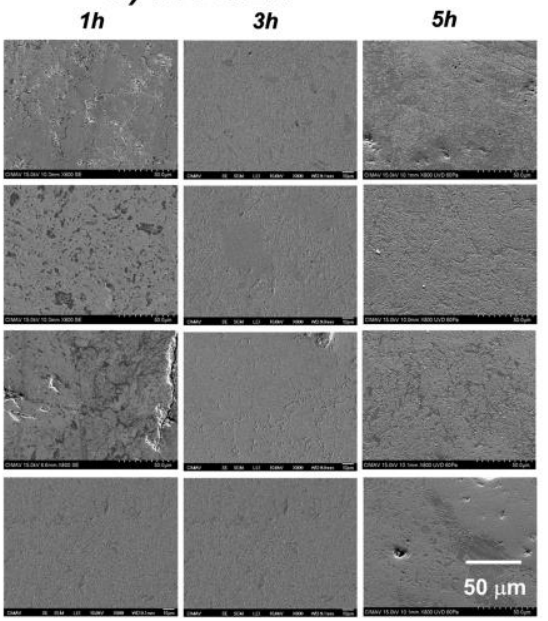

c) Densification
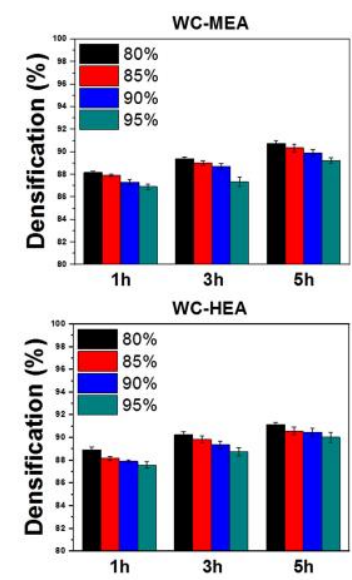

Figure 1. SEM-SE micrographs corresponding to a)WC-MEA and b)WC-HEA with variations in the sintering time and concentration of $\mathrm{WC}$, and c) densification of the sintered samples.
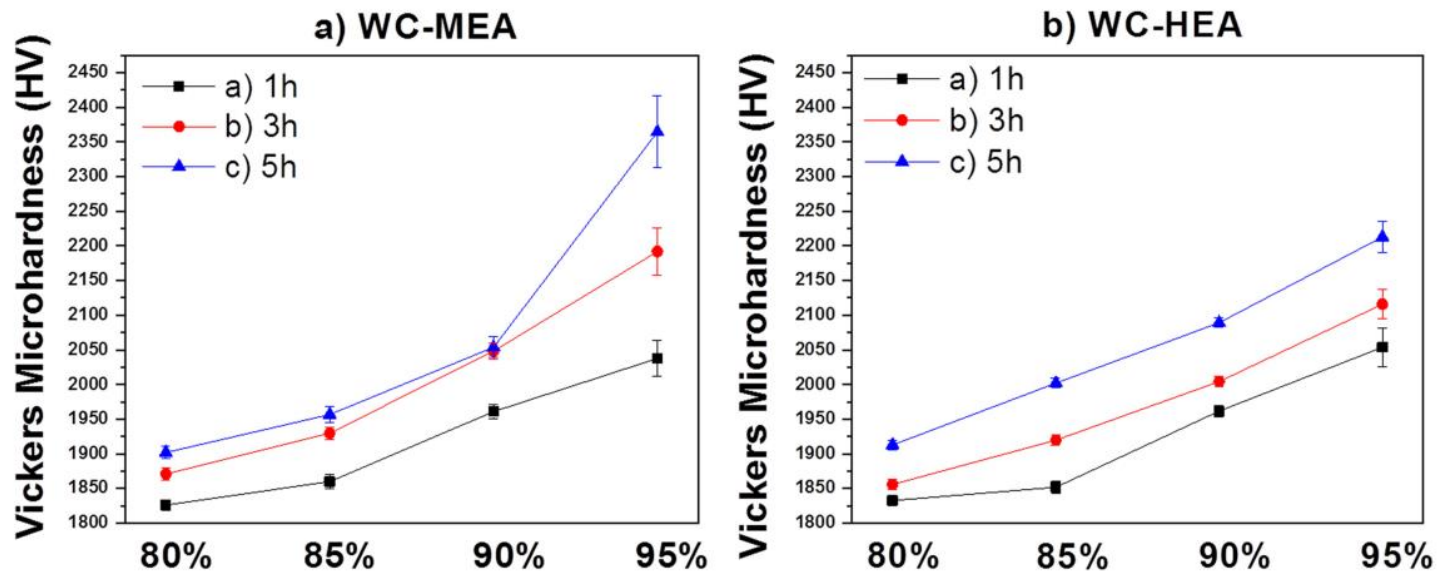

Figure 2. Vickers microhardness values of the sintered samples, a) WC-MEA and b) WC-HEA, with variations in the sintering time and concentration of WC.

\section{References}

[1] Lining G, Jiwu H, \& Chen X, Effects of carbon content on microstructure and properties of WC-20Co cemented carbides, (2014), International Journal of Refractory Metals and Hard Materials, Volume 42, p. 228-232.

[2] Miracle D.B., Senkov O.N., A critical review of high entropy alloys and related concepts, (2017) Acta Materialia, Volume 122, p. 448-511. 